\section{News for 2015}

\section{Domenico Prisco ${ }^{1}$}

Published online: 8 July 2015

(C) SIMI 2015

\section{Dear Readers,}

I am proud to announce that Internal and Emergency Medicine has received a very respectable Impact Factor (IF), 2.624, which is another improvement over the factors of 2.41, 2.345 and 2.057, respectively, obtained for 2013, 2012 and 2011. During 2014, the journal received 412 citations for the 157 articles published in 2012 and 2013. Our Journal is ranked 33 out of 153 journals (Q1) in the category Medicine General and Internal.

About the news of 2015, I underline that we have caught up with the backlog, and the insertion of papers into a specific issue is more rapid. Please note that the Clinical Evidence section with Cochrane Corner and HTA has been enriched with interesting papers thanks to the excellent work of Giorgio Costantino and Giovanni Casazza. Moreover, all is doing well with Emergency section now headed by Bruce Adams after the tremendous work of Peter Rosen done over the last 9 years. Peter is still working for IEM by selecting interesting Medical Illustrations among the numerous proposals we receive every week.

As usual I warmly thank all the members of different boards, the president and the council of SIMI and the publisher Springer. Last not least thanks to our readership for whom creating this journal is a great fulfilment.

Domenico Prisco, Editor in Chief

Domenico Prisco

domenico.prisco@unifi.it

1 Florence, Italy 